\title{
MicroRNA hsa-miR-135b Regulates Mineralization in Osteogenic Differentiation of Human Unrestricted Somatic Stem Cells
}

\author{
Aneta M. Schaap-Oziemlak, ${ }^{1}$ Reinier A. Raymakers, ${ }^{2}$ Saskia M. Bergevoet, ${ }^{1}$ Christian Gilissen, ${ }^{3}$ \\ Bastiaan J.H. Jansen, ${ }^{4}$ Gosse J. Adema, ${ }^{4}$ Gesine Kögler, ${ }^{5}$ Carlos le Sage,${ }^{6}$ Reuven Agami, ${ }^{6}$ \\ Bert A. van der Reijden, ${ }^{1}$ and Joop H. Jansen ${ }^{1}$
}

Unrestricted somatic stem cells (USSCs) have been recently identified in human umbilical cord blood and have been shown to differentiate into lineages representing all 3 germ layers. To characterize microRNAs that may regulate osteogenic differentiation of USSCs, we carried out expression analysis for 157 microRNAs using quantitative RT-PCR before and after osteogenic induction $(t=0.5,24,72,168,216 \mathrm{~h})$. Three microRNAs, hsa-miR-135b, hsa-miR-224, and hsa-miR-31, were consistently down-regulated during osteogenesis of USSC line 1. Hsa-miR$135 \mathrm{~b}$ was shown to be the most profoundly down-regulated in osteogenesis of USSC line 1 and further confirmed to be down-regulated in the osteogenic differentiation of 2 additional USSC lines. Function of hsa-miR-135b in osteogenesis of USSCs was examined by retroviral overexpression, which resulted in an evident decreased mineralization, indicating that hsa-miR-135b down-regulation is functionally important for full osteogenic differentiation of USSCs. MicroRNAs have been shown to regulate negatively expression of their target gene(s). To identify putative targets of hsa-miR-135b, we performed cDNA microarray expression analysis. We selected in total 10 transcripts that were down-regulated ( $\geq 2$-fold) in response to hsa-miR-135b overexpression at day 7 and day 9 of osteogenic differentiation. The function of most of these targets in human osteogenesis is unknown and requires further investigation. Markedly, quantitative RT-PCR data showed decreased expression of osteogenic markers IBSP and Osterix, both known to be involved in bone mineralization, in osteogenesis of USSCs that overexpress hsa-miR-135b. This finding suggests that hsa-miR-135b may control osteoblastic differentiation of USSCs by regulating expression of bone-related genes.

\section{Introduction}

$\mathrm{M}$ ICRORNAS REPRESENT A LARGE and expanding class of small, single-stranded, non-coding RNAs found in both the plant and animal kingdoms. The 19-25 nucleotides long mature microRNAs are generated from endogenous double-stranded transcripts containing hairpin structures by the action of multiprotein complexes involving RNAse-III-like endonucleases (Drosha and Dicer) (ref. 1; reviewed in [2,3]). MicroRNAs have been shown to play roles in diverse biological processes, including proliferation, differentiation, and apoptosis (reviewed in ref. [4-6]). MicroRNAs exert their biological function by negative regulation of the expression of target genes, either by mRNA degradation and/or translational repression, depending respectively on perfect or imperfect complementarity of the mature microRNA sequence to the 3'- or $5^{\prime}$-UTRs of the target microRNAs (ref. 7; reviewed in ref. $[6,8])$. To identify possible target mRNAs for any given microRNA, publically available computational prediction algorithms such as miRBase (ref. 9; www.microrna.sanger. ac.uk), PicTar (ref. 10; www.pictar.bio.nyu.edu), DIANAmicroT (ref. 11; www.diana.pcbi.upenn.edu/cgi-bin/micro_t. cgi), and TargetScanS (ref. 12; www.genes.mit.edu/tscan/ targetscanS) have been developed.

${ }^{1}$ Central Hematology Laboratory, ${ }^{2}$ Department of Hematology, ${ }^{3}$ Department of Human Genetics, ${ }^{4}$ Department of Tumor Immunology, Radboud University Nijmegen Medical Centre, Nijmegen Center for Molecular Life Sciences, Nijmegen, The Netherlands.

${ }^{5}$ Institute for Transplantation Diagnostics and Cell Therapeutics, University of Düsseldorf Medical School, Düsseldorf, Germany.

${ }^{6}$ Division of Gene Regulation, The Netherlands Cancer Institute, Amsterdam, The Netherlands. 
To determine the role of microRNAs in the regulation of osteogenic differentiation of human pluripotent stem cells, we investigated the osteogenic differentiation potential of the recently described unrestricted somatic stem cells (USSCs), derived from umbilical cord blood [13]. This neonatal type of stem cells with spindle-shaped morphology has been shown to give rise to different lineages including osteogenic, chondrogenic, adipogenic, hepatogenic, neurogenic, and hematopoietic [13-15].

Osteogenesis is described as a well-orchestrated multistep process, in which multipotent stem cells give rise to committed osteoprogenitors that in turn differentiate into osteoblasts. These undergo further maturation by production of bone matrix proteins, followed by the extracellular matrix (ECM) mineralization (ref. [16]; reviewed in ref. [17]). Molecular pathways underlying this differentiation program have not yet been entirely examined. Specifically, a thorough expression analysis of microRNAs combined with the experimental examination of their function and target genes in the osteogenesis of USSCs has never been described. Functional validation of most differentially expressed microRNA in the osteogenic differentiation of USSCs is important to understand the regulatory mechanisms of microRNA(s) in the osteogenic differentiation of stem cells.

\section{Materials and Methods}

\section{Generation and culture of USSC lines}

USSCs used in all experiments were generated from fresh umbilical cord blood as described before [13]. In brief, the mononuclear fraction of cells obtained by a Ficoll (density $1.077 \mathrm{~g} / \mathrm{cm}^{3}$; Ficoll-paque 1077; GE Healthcare, Uppsala, Sweden) gradient centrifugation was plated for 2 weeks in USSC proliferation medium (DMEM-LG [Lonza, Verviers, Belgium] supplemented with preselected FBS (30\%; [HyClone, South Logan, Utah]), penicillin (100 U/mL) and streptomycin $(0.1 \mathrm{mg} / \mathrm{mL}$; [MP Biomedicals Inc., Cleveland, $\mathrm{OH}])$, ultraglutamine (2 mM; [Cambrex, Verviers, Belgium]), and dexamethasone $\left(10^{-7} \mathrm{M}\right.$; [Sigma-Aldrich Inc., St. Louis, $\mathrm{MO}])$ ). After 2 weeks, cells were cultured for an additional 2-4 weeks without dexamethasone until adherent colonies were observed. Expansion of these colonies was performed by 5 subsequent passages, followed by freezing the cells and thereby establishing the USSC lines.

USSC lines were cultured in proliferation medium at $37^{\circ} \mathrm{C}$ and $5 \% \mathrm{CO}_{2}$ in a humidified atmosphere. At $70 \%$ confluency, cells were trypsinized and replated in the volume ratio 1:3.

\section{Osteogenic differentiation of USSCs in vitro}

USSCs (passages 9-13), derived from 3 different donors, were plated at a cell density of 8,000 or 16,000 cells $/ \mathrm{cm}^{2}$ in 24-well plates or 6-well plates (as indicated in text). At $70 \%$ confluency, proliferation medium was replaced by osteogenic medium containing dexamethasone $\left(10^{-7} \mathrm{M}\right)$, ascorbic acid (50 $\mu \mathrm{M}$; Sigma-Aldrich, St. Louis, MO), and $\beta$-glycerophosphate (10 mM; MP Biomedicals Inc., Solon, $\mathrm{OH})$ as previously described [13]. USSCs were differentiated for 14 days. Alkaline phosphatase (ALP) activity was tested at different time points (0,3, 7, 9, and 14 days) during USSCs osteogenesis by performing ALP staining using as substrate a naphthol AS-MX phosphate (Sigma-Aldrich,
St. Louis, MO). To quantify ALP activity, $p N P P$ ( $p$-nitrophenylphosphate) hydrolysis method was applied, followed by reading the absorbance at $405 \mathrm{~nm}$ in duplicate (empty vector and overexpressing hsa-miR-135b) in 96-well format. A background absorbance of empty wells was subtracted from the absorbance of the samples.

To test mineralization, Alizarin Red S (ARS) staining was performed, where cells were first washed twice with PBS, then fixed with $4 \%$ paraformaldehyde, washed with water, and incubated with ARS solution $(1 \%, \mathrm{pH}=4.1$; Sigma-Aldrich, Buchs, UK) for $5 \mathrm{~min}$ at room temperature, followed by 3 washings with water while gently shaking for $5 \mathrm{~min}$.

\section{Alizarin Red S quantification}

Quantification of the mineralization at different time points during USSCs osteogenesis was based on adapted protocol described by Gregory et al. [18]. In brief, for quantification ARS staining (40 $\mathrm{mM}$ solution, $\mathrm{pH}=4.1$ ) was performed on USSCs and differentiated (osteogenic; initial seeding density 16,000 cells/ $\mathrm{cm}^{2}$ ) monolayers in 6-well plates for $30 \mathrm{~min}$ at room temperature with gentle shaking, followed by washing with water and storage of plates at $-20^{\circ} \mathrm{C}$ before ARS quantification. ARS was extracted from the stained monolayer by incubation with $10 \%$ (v/v) acetic acid (0.8 mL/well; Sigma-Aldrich, St. Louis, MO) while shaking, followed by scraping the cells and transfer of the slurry to a $1.5-\mathrm{mL}$ Eppendorf tube and overlaying it with mineral oil (Sigma-Aldrich). The slurry was then heated to $85^{\circ} \mathrm{C}$ for $10 \mathrm{~min}$, transferred on ice, and centrifuged at $15,000 \mathrm{~g}$ for $15 \mathrm{~min}$ at room temperature. Next, $0.2 \mathrm{~mL}$ ammonium hydroxide (10\% (v/v); Sigma-Aldrich) was added to $0.5 \mathrm{~mL}$ of supernatant to neutralize the acid. The extracted ARS was quantified by reading the absorbance at $405 \mathrm{~nm}$ in duplicate (empty vector) or quadruplicate (overexpressing hsa-miR$135 b)$ of supernatant aliquots $(150 \mu \mathrm{L})$ in 96-well format. A background absorbance of empty wells was subtracted from the absorbance of the samples.

\section{Retroviral vectors and virus production}

Phoenix amphotrophic ( $\Phi-\mathrm{NX}-\mathrm{A})$ virus producer cell line (a gift from Dr. GP Nolan, Stanford University Medical Center, Stanford, CA) was used to generate retroviral vector particles carrying constructs encoding microRNA minigenes or control vectors (empty miR-Vec-Ctrl and miRVec-YFP). All retroviral constructs used in this study were kindly provided by Dr. Reuven Agami (The Netherlands Cancer Institute, Amsterdam), and their generation was described in detail by Voorhoeve et al. [19]. In brief, miRVec-Ctrl and microRNA minigene containing vectors were based on the pMSCV-puro (Clontech, Mountain View, CA) backbone, where PGK-promoter and puromycin-resistant gene were replaced, respectively, by CMV promoter and the blasticidin-resistant gene [19]. The miR-Vec-EYFP, in which the blasticidin-resistant gene was replaced by EYFP (pEYFP-N1; Clontech), was used as a control to test transduction efficiency in USSCs. The miR-Vec-Ctrl was included as an empty vector control in hsa-miR-135b overexpression experiments.

Transfection of all retroviral constructs into $\Phi-\mathrm{NX}-\mathrm{A}$ producer line was performed by using the calcium phosphate 
method. Briefly, $24 \mathrm{~h}$ prior transfection $\Phi-\mathrm{NX}$-A cells were plated at $2.8 \times 10^{6}$ cells $/ 75 \mathrm{~cm}^{2}$. Sixteen hours after transfection, the $\Phi-\mathrm{NX}$-A culture medium [DMEM high-glucose medium (Gibco, Paisley, Scotland, UK), supplemented with FCS (10\%; Gibco), penicillin (100 U/mL), streptomycin $(0.1 \mathrm{mg} /$ mL; MP Biomedicals Inc., Solon, $\mathrm{OH}$ ), and L-glutamine (200 mM; MP Biomedicals Inc.)], was refreshed. Twenty-four and $48 \mathrm{hrs}$ after medium refreshment, the medium containing retroviral particles was collected, filtered through a sterile $0.45-\mu \mathrm{m}$ pore size filter (Whatman $\mathrm{GmbH}$, Dassel, Germany), and stored as aliquots at $-80^{\circ} \mathrm{C}$.

\section{USSCS retroviral transduction}

Before retroviral transduction, 35-mm Petri dishes were coated with retronectin (Takara Bio Inc., Otsu, Shiga, Japan). $0.4 \times 10^{6}$ USSCs (line 1; passages 8 and 9) after 1-h incubation with viral supernatant at $37^{\circ} \mathrm{C}$ were plated into viruspreloaded retronectin-coated 35-mm Petri dishes $\left(0.2 \times 10^{6}\right.$ USSCs/dish) and further incubated with the virus for 24-48 $\mathrm{h}$ at $37^{\circ} \mathrm{C}$ and $5 \% \mathrm{CO}_{2}$ in a humidified atmosphere. Next, USSCs were washed twice with Hank's balanced salt solution (HBBS; Lonza, Verviers, Belgium) and after 24-48 h transduced USSCs were selected by culturing them in USSC proliferation medium in the presence of blasticidin ( $5 \mu \mathrm{g} /$ $\mathrm{mL}$; Invitrogen Corporation, Carlsbad, CA) for 7 days. In parallel, to test the blasticidin toxicity, a selection control (not transduced USSCs) was included, where 100\% of USSCs died after 1-2 days of exposure to the blasticidin. After blasticidin selection, transduced USSCs were expanded for 2-3 days prior to osteogenic differentiation.

Transduction efficiency of USSCs with usage of the miRVec-EYFP construct, measured by EYFP expression by flow cytometry (Beckman Coulter Cytomics FC 5000; Beckman Coulter, Miami, FL), was in a range of $12 \%-22 \%$.

\section{Total RNA isolation}

Total RNA was isolated from undifferentiated and differentiated USSCs either by using the RNA-Bee isolation kit (Iso-Tex Diagnostics, Friendswood, TX) or the miRVana isolation kit (Ambion Inc., Austin, TX) according to the manufacturer's protocols. For harvesting, an adherent layer of USSCs was first washed with PBS, followed by addition of lysis buffer and scraping. The RNA concentration was measured by ND-1000 spectrophotometer (NanoDrop Technologies, Wilmington, DE) and the quality of RNA was estimated by electrophoresis based on the integrity of rRNA (18S and 28S).

\section{Expression analysis}

cDNAs, used as templates in RT-PCR, from undifferentiated and differentiated USSCs were obtained by performing a RT reaction, in which $1 \mu \mathrm{g}$ of total RNA was used. For a relative (normalized to GAPDH) expression analysis, a real-time reverse transcription (RT)-PCR was performed (ABI/PRISM 7700 Sequence Detection System; ABI/PE, Foster City, CA) by using SYBR Green PCR master mix (Applied Biosystems, Foster City, CA) and primers specific for: RUNX2/CBFA1, forward, 5'-GCAAGGTTCAACGATCTGAGA-3', reverse, 5'-TCCCCGAGGTCCATCTACTG-3' (141 bp); osteocalcin/
BGLAP, forward, 5'-AGCAAAGGTGCAGCCTTTGT-3', reverse, 5'-GCGCCTGGGTCTCTTCACT-3' (63 bp); OSX/ SP7 (osterix), forward, 5'-GTGGGCAGCTAGAAGG GAGT-3', reverse, 5'-AATTAGGGCAGTCGCAGGA-3' (114 $\mathrm{bp);} \mathrm{IBSP} \mathrm{(bone} \mathrm{sialoprotein),} \mathrm{forward,} \mathrm{5'-CATAAACGG}$ CACCAGTACCAA-3', reverse, 5'-CTCCATTGTCTCCT CCGCT-3' (71 bp). For a quantitative analysis of expression level of mature microRNAs, the microRNA human panel expression assay (Early Access Kit PN4365381; Applied Biosystems; Supplementary Table 1; Supplementary materials are available online at www.liebertonline.com/scd) was applied. RNA input was normalized by GAPDH RT-PCR (TaqMan Gene Expression Assay for human GAPDH; Applied Biosystems). First each microRNA-specific RT reaction with a specific stem-loop RT primer was performed using $10 \mathrm{ng}$ of total RNA, followed by a real-time RT-PCR with $1.3 \mu \mathrm{L}$ of RT-product, microRNA-specific tailed forward primer, and nonspecific microRNA reverse primer as previously described [20].

\section{Microarray data generation and analysis}

For microarray experiments, total RNA from 2 undifferentiated USSCs (line 1; passages 11 and 12) and differentiated from day 7 and day 9 of osteogenesis, including empty vector-transduced- and hsa-miR-135b-overexpressing USSCs, was isolated using the RNeasy Mini kit (Qiagen) according to the manufacturer's protocol. Quality of RNA was tested using the Agilent 2100 Bioanalyzer. One hundred nanograms of total RNA from each sample were used for cDNA amplification and $5.5 \mu \mathrm{g}$ of amplified cDNA of each sample was applied to perform hybridizations on the GeneChip Human Exon 1.0 ST Array (Affymetrix) according to the Affymetrix GeneChip Expression Analysis Technical Manual (GeneChip ${ }^{\circledR}$ Whole Transcript (WT) Sense Target Labeling Assay Manual P/N 701880 Rev. 4, www.affymetrix. com). This human exon array contains $>1,400,000$ probe sets with an average of 4 probes per exon and about 40 probes per gene (Affymetrix Technical Note, www.affymetrix.com/ support/technical/datasheets/exon_arraydesign_datasheet. pdf, Part No. 702026 Rev. 1). Genomic positions of the probe sets for each transcript (so called transcript cluster ID) given in the article can be found online by using The NetAff $x^{\mathrm{TM}}$ Analysis Center (www.affymetrix.com/analysis/index. affx).

Gene level expression data were calculated for the CORE transcripts using Affymetrix Expression Console software using sketch-quantile normalization, PM-GCBG background correction, and summarization with the PLIER algorithm. Pearson's correlation was used to check correspondence between the samples. Data were then imported into GeneSpring GX 7.3, where per array the expression of each transcript was normalized to the median. Genes with a $\geq 2$-fold change between the samples were selected.

\section{Statistical analysis}

All included data are presented as a mean with standard error shown. For determining $P$ value, unpaired (different USSC lines) or paired (the same USSC line) Student's $t$-test for 2 tails was performed. $P$ value $\leq 0.05$ (marked as "a") was defined as statistically significant. 


\section{Results}

\section{Expression profile of microRNAs in osteogenic differentiation of USSCs}

Culturing of USSC lines in low-glucose-containing medium in the presence of osteogenic inductive factors (dexamethasone, $\beta$-glycerophosphate, and ascorbic acid) led to a typical osteogenic phenotype: a cuboidal cell morphology, characteristic for osteoblasts, increased activity of the ALP, and mineralization of the ECM (reviewed in ref. [17,21]). An increase in both the number of ALP-positive (blue) cells and ALP activity per cell was observed starting from day 3 until day 7 of osteogenesis, reaching a peak of the ALP activity, characteristic for the osteoblastic stage, at day 7 (Fig. 1A). Following day 9 of the osteogenic differentiation of USSCs, the ALP activity decreased up to day 14 . The degree of mineralization was evaluated based on ARS staining and examined at different time points during osteogenesis: days 0, 3, 7, 9, and 14 (Fig. 1B). At day 7 the first mineral deposits appeared (orange staining), followed by more robust mineralization at day 9 and day 14 of differentiation. Furthermore, a significantly elevated expression of osteogenic markers such as ALP, Runx2, collagen type I, and osteocalcin confirmed osteogenic differentiation potential of the USSCs (data not shown).

To identify microRNAs involved in the regulation of the osteogenic differentiation of USSCs, we measured the expression of 157 microRNAs by a real-time RT-PCR at different time points of osteogenesis $(0,0.5,24,72,168$, and $216 \mathrm{~h})$ of USSC line 1 . We found that 3 microRNAs, hsa-miR-135b, hsa-miR-224, and hsa-miR-31, were consistently down-regulated between day 3 and day 9 of the osteogenic differentiation of USSC line 1. The expression level of hsa-miR-135b was decreased 100-fold at day 9 compared to undifferentiated USSCs and expression of hsa-miR-31 and hsa-miR-224 declined at day 9, respectively, by a factor 14.3 and 20 (Fig. 1C). Since hsa-miR-135b was the most profoundly downregulated microRNA in osteogenesis of USSC line 1, we examined its expression during osteogenesis in 2 additional USSC lines, derived from 2 different donors. Similarly to USSC line 1, we observed a decrease in the expression level of this microRNA during the osteogenic differentiation of USSC lines 2 and 3, albeit to a lower degree (data not shown). Combining the expression data of 3 biological replicates of hsa-mir-135b in osteogenesis, a 4.2-fold and a 2.8-fold decreased expression of this microRNA, respectively, at day 7 and day 9 of the osteogenic differentiation was observed.

\section{Function of hsa-miR-135b in osteogenic differentiation of USSCs}

It has been shown that inhibition of microRNA(s) during osteogenic differentiation appears to be necessary to release the repression of genes essential for driving the osteogenic differentiation program [22]. Therefore, we decided to investigate the function in the osteogenic differentiation of USSCs of most down-regulated microRNA, hsa-miR-135b. We retrovirally overexpressed hsa-miR-135b in USSCs and induced osteogenic differentiation. Overexpression of hsa-miR-135b ( $>500$-fold) was confirmed by real-time RT-PCR both in undifferentiated USSCs and osteogenic cells (Fig. 2B). A profound decrease in ARS staining was observed at day 7 during the osteogenic differentiation of USSCs overexpressing hsamiR-135b compared to empty vector-transduced USSCs (Fig. 2A). In addition, the quantitative measurement of the ARS staining at different time points during osteogenesis showed reduced ARS staining by 2.7 -fold at day 7 , followed by 1.5 fold at day $8,1.7$-fold at day $9,1.6$-fold at day 12 , and 2.2-fold at day 14 relative to the empty vector control (Fig. 2C). In parallel to the ARS staining, we performed ALP staining during the osteogenic differentiation but we did not observe any significant changes in the ALP activity of hsa-miR-135b-overexpressing USSCs compared to empty vector controltransduced USSCs (Supplementary Fig. 1).

\section{Discussion}

In this study, we showed that among 157 human microRNAs tested, hsa-miR-135b was the most profoundly downregulated microRNA during osteogenesis of USSC line 1. This down-regulation was further confirmed in osteogenesis of 2 additional independent USSC lines. A recently published microRNA expression profiling of osteogenesis of murine mesenchymal $\mathrm{C} 2 \mathrm{C} 12$ cells showed that most $(88 \%)$ of the differentially expressed microRNAs in response to BMP2-induced osteogenesis were down-regulated [22]. These results are consistent with the osteogenic signature of USSCs presented in our study since we found mainly downregulated microRNAs during the osteogenesis of USSCs. In addition, one of the functionally important microRNAs, down-regulated in $\mathrm{C} 2 \mathrm{C} 12$ osteogenesis, appeared to be miR-135a [22], a family member of miR-135b. Importantly, regarding sequence conservation, both miR-135a and miR$135 \mathrm{~b}$, are identical between human and mouse.

Notably, we did not find any microRNAs that were consistently up-regulated during the osteogenic differentiation of USSCs including let-7a that has been shown previously to be up-regulated (at day 7) in this process [23]. Furthermore, the down-regulation of hsa-miR-135b during osteogenesis observed in this study was not observed in the study by Landgraf et al., in which hsa-miR-135b was undetectable before and after the osteogenic induction. These differences in the microRNA expression profiling between our data and the data by Landgraf et al. may be explained by the use of various detection methods, which differ both in sensitivity and specificity.

FIG. 1. Osteogenic differentiation of unrestricted somatic stem cells (USSCs) and differentially expressed microRNAs. (A) ALP (alkaline phosphatase) assay performed during different time points in USSCs osteogenesis, showing an increase in ALP activity (blue) with a peak at day 7; magnification 5×. (B) Mineralization was detected by performing Alizarin Red S (ARS) staining at different time points in osteogenesis; magnification $5 \times$. (C) Expression of microRNAs in osteogenesis of USSC line 1 shown as a logarithmic graph with indicated relative (to $t=0$ ) expression level with standard error. Data calculated based on averages of 2 amplifications, followed by calculating relative to $t=0$ expression per each time point. Statistically significant data, relative to $t=0$ shown with $P$ value $\leq 0.05$ (marked as "a"). Each photo in (A) and (B) represents two-third of the well. 

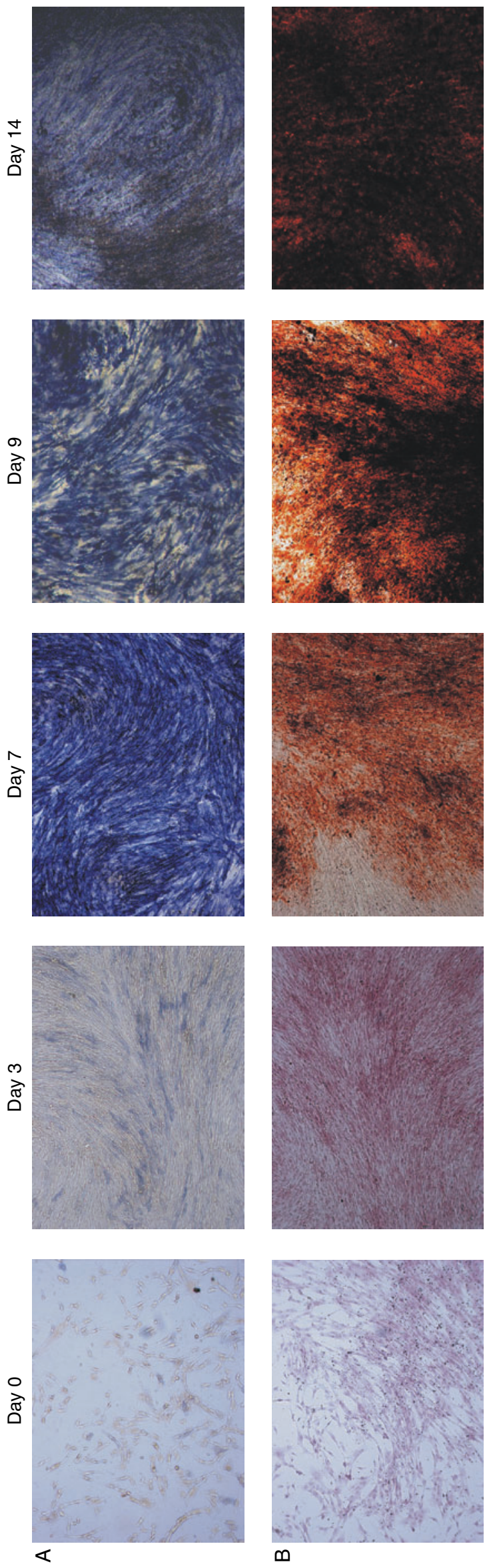

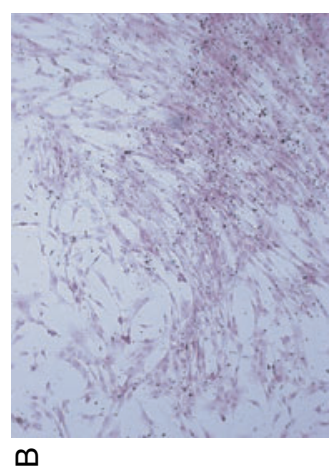

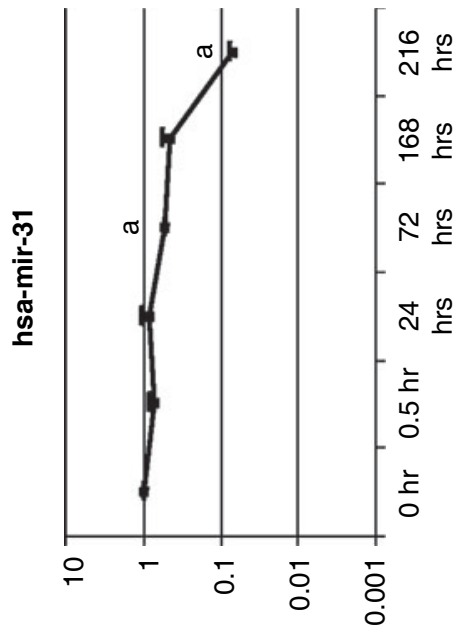

uо!़səлdxә әм!̣е|әу

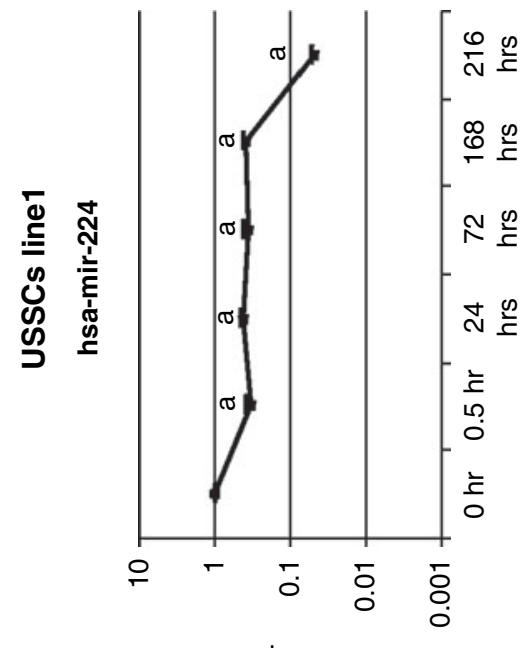

uo!ssəıdxә әм!ฺе|әу

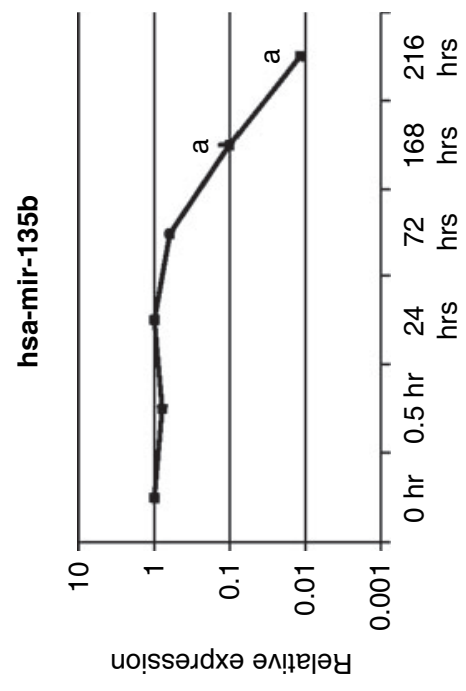


Considering the fact that the most significant down-regulation of hsa-miR-135b was observed during the osteogenic differentiation of all 3 USSC lines between day 7 and day 9 may point to an important role of hsa-miR-135b in mineralization. Indeed, in our study overexpression of hsa-miR-135b in USSCs osteogenesis led to an evident decrease (2-fold in average) in the level of ARS staining within this particular time frame of differentiation. Furthermore, based on our data showing a decrease in mineralization as a result of deregulated hsa-miR-135b expression levels, and data of others showing a robust inhibition of mineralization upon knockdown of the microRNA-processing enzyme Dicer using human mesenchymal stem cells [24], the proper expression level of microRNA(s) during osteogenesis is critical for the full osteogenic differentiation potential.

The lack of any significant changes in the ALP activity during the osteogenic differentiation of hsa-miR-135boverexpressing USSCs suggests that the induction of this enzyme alone, known also as TNAP (tissue-nonspecific ALP; reviewed in ref. [17]), is not sufficient for the mineralization process. This observation might be in line with a debatable solitary role of TNAP in the mineral deposition (reviewed in ref. [25-30]). From mouse knockout studies, it has been shown that the TNAP deficiency results in a normal embryonic skeletal development and poor mineralization at postnatal stages [27,31], suggesting that TNAP does not play an essential role in the initiation of mineralization but is rather important for its maintenance.

The down-regulation of hsa-miR-135b during USSCs osteogenesis might also be compatible with a role in maintenance of the stemness of USSCs. Interestingly, recently published data from embryonic stem (ES) cells have shown a conserved (both in mouse and human) binding of Oct4, one of the key regulators of self-renewal and pluripotency of ES cells, at the promoter of the $m i R-135 b$ gene [27], suggesting that $m i R-135 b$ gene is a direct target of Oct 4 in ES cells.

The identification of hsa-miR-135b helps to elucidate the basic regulatory network implicated in human osteogenic differentiation. Furthermore, to understand the molecular mechanism underlying hsa-miR-135b function in USSCs osteogenesis, we performed both an expression analysis of osteoblast markers such as osteocalcin (BGLAP), bone sialoprotein (IBSP), osterix (OSX), and RUNX2 by real-time RT-PCR, and a microarray analysis for undifferentiated and differentiated USSCs overexpressing hsa-miR-135b and empty vector control at days 0,7 , and 9 of osteogenesis in the same time-course experiment. In contrast to the expression of BGLAP and RUNX2, which remained unchanged, the expression of bone sialoprotein (IBSP) and OSX was decreased at day 7 by 7.18-fold and 3.18-fold, respectively, and at day 9 by 3.56-fold and 1.55-fold, respectively, in hsamiR-135b-overexpressing USSCs compared to empty vector-transduced USSCs (Fig. 3). Though both IBSP and OSX do not represent direct computational target genes of hsamiR-135b, they have been shown to be functionally important in ossification. The role of IBSP particularly in de novo bone mineralization has been reported before [32,33], and strongly suggests that its decreased expression level at day 7 and day 9 in hsa-miR-135b-overexpressing USSCs might be responsible for the observed decline in the mineralization.

To further dissect the possible mechanism(s) of hsa-miR$135 \mathrm{~b}$ action in USSCs osteogenesis, we decided to identify putative target genes of hsa-miR-135b. At this point, it is important to underline that microRNAs may affect expression of their targets both at the transcriptional as well as at the translational level (reviewed in ref. $[35,36]$ ). In addition, although most of the parameters used by target prediction algorithms are similar (complementarity of $5^{\prime}$ sequence of microRNA to 3' UTR sequences of target mRNA(s), phylogenetic sequence conservation of $3^{\prime}$ UTRs or mRNA(s) target multiplicity), each of these programs varies substantially by types of predicted targets, the numbers of covered genomes, followed by differences in percentages of false-positive rates (reviewed in ref. [8]). Therefore, an experimental validation of computational mRNA targets for specific microRNA is always necessary. In our study, the selection of hsa-miR-135b targets was based on the changes at the transcriptional level of the target gene(s). First, we performed a microarray-based gene expression analysis of 2 undifferentiated USSCs: empty vector-transduced USSC s and hsa-miR-135b-overexpressing USSCs, followed by a microarray-based gene expression analysis of differentiated USSC at day 7 and day 9 of osteogenesis. Taking into account the time kinetics of hsa-miR-135b down-regulation in USSCs osteogenesis, we selected in total 10 transcripts that were down-regulated ( $\geq 2$-fold) in response to the hsa-miR-135b overexpression at day 7 and day 9 of osteogenic differentiation (Supplementary Table 2). These genes did not appear to be in silico targets as they were not identified by miRBase or TargetScan. The function of 7 of these target genes, which included IL31, KCQIDN, MGC23284, REX01L1, CRIP1, CKB, and $L H B$, in human osteogenesis remains unknown and should be further investigated. The other 2 experimentally identified

FIG. 2. Hsa-miR-135b overexpression in unrestricted somatic stem cells (USSCs) osteogenesis. (A) To monitor the mineral deposition, Alizarin Red S (ARS) staining was performed at indicated different time points during the osteogenic differentiation of USSCs overexpressing hsa-miR-135b (bottom panel) and USSCs transduced with empty vector as a control (upper panel); magnification $5 \times$ and each photo represents two-third of the well. The data shown here represent 2 separate differentiation experiments that yielded similar results. (B) The expression of hsa-miR-135b during osteogenesis of USSCs (line 1) was measured by a real-time RT-PCR at indicated time points. The expression level of hsa-miR-135b in both untransduced USSCs and overexpressing hsa-miR-135b is represented by the absolute Ct values. Hsa-miR-135b = expression in untransduced USSCs; OV (24 h) hsa-miR-135b = hsa-miR-135b-overexpressing USSCs (USSCs incubated with virus for 24 h); OV (48 h) hsa-miR-135b = hsa-miR-135b-overexpressing USSCs (USSCs incubated with virus for $48 \mathrm{~h}$ ). Data were calculated based on averages of 2 amplifications per each condition, with standard error shown. Statistically significant data, relative to $t=0$ shown with $P$ value $\leq 0.05$ (marked as "a"). (C) Mineralization determined by a spectrophotometric measurement of extracted ARS dye at indicated time points. OS = USSCs induced toward osteogenic lineage; UN = USSCs cultured in undifferentiated condition; hsa-miR-135b = hsa-miR-135b-overexpressing USSCs. Data represent mean absorbance at $405 \mathrm{~nm}$ with standard error shown. Statistically significant data, relative to $t=0$ of each condition shown with $P$ value $\leq 0.05$ (marked as "a"). 

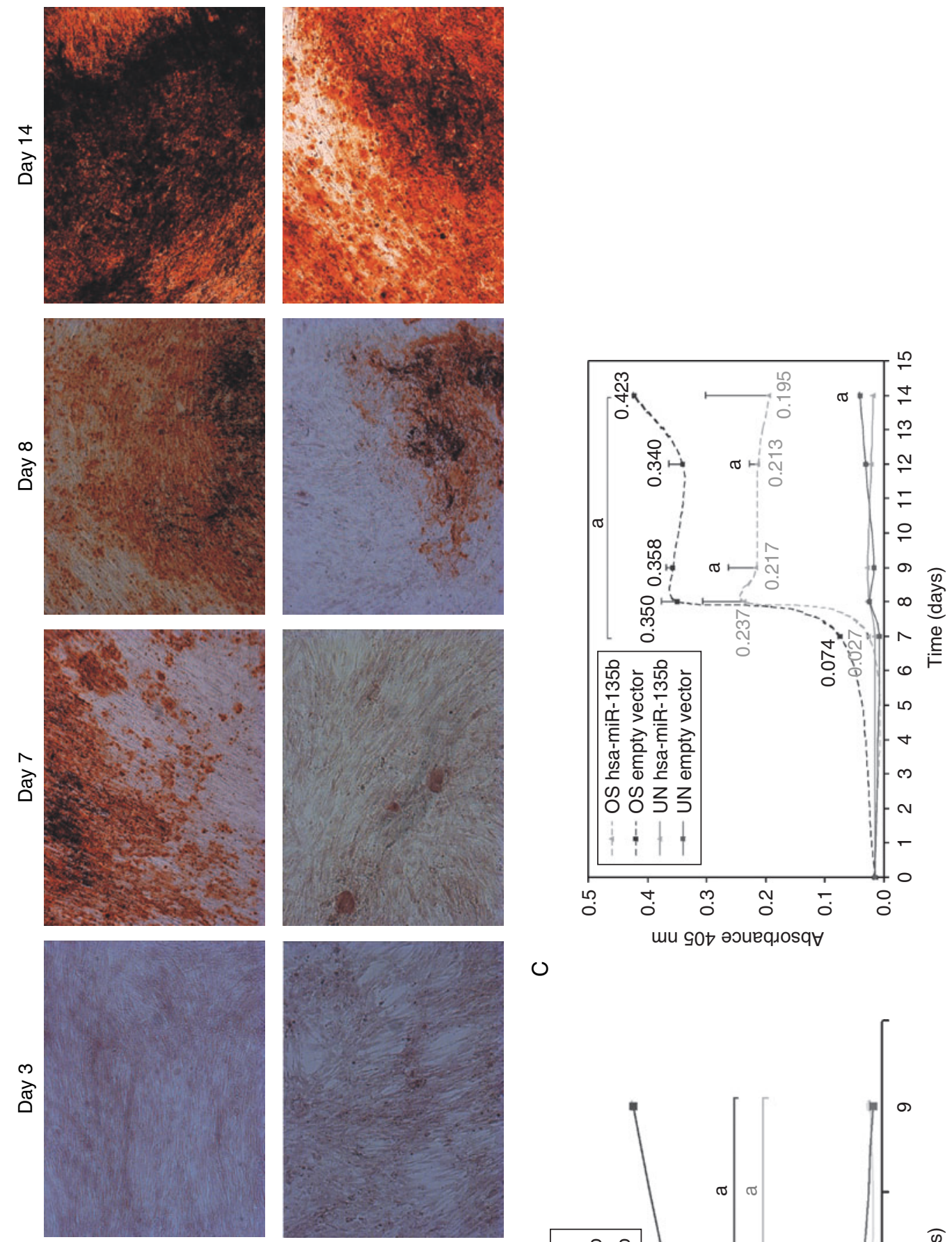

0

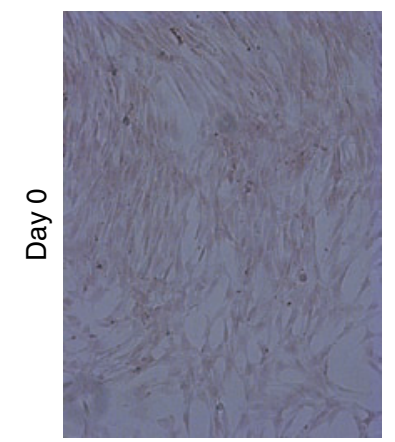

竞高

$\varangle$

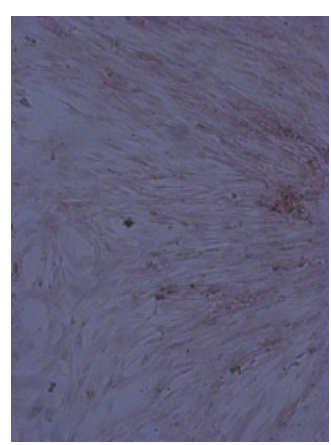

$\frac{\text { 율 }}{\frac{m}{\dot{1}}}$

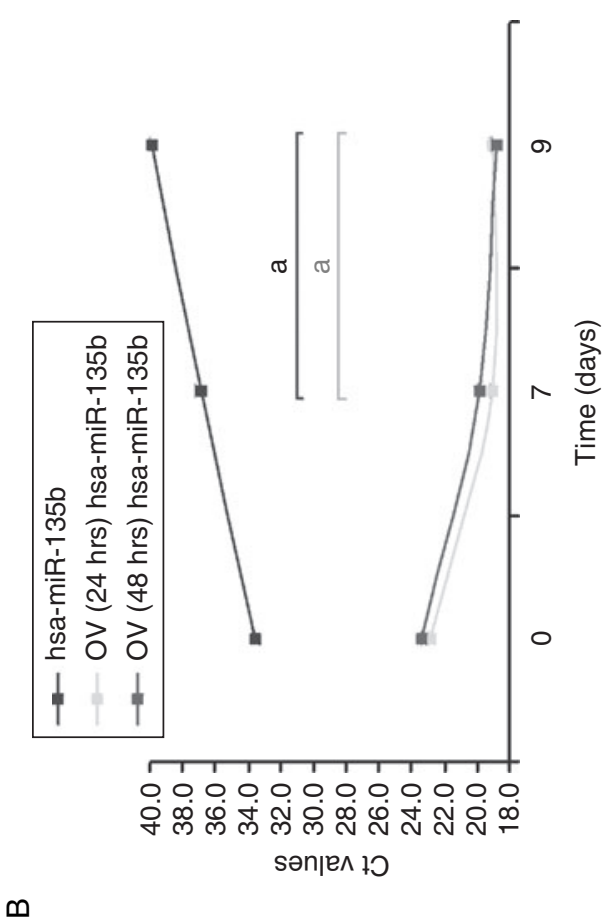



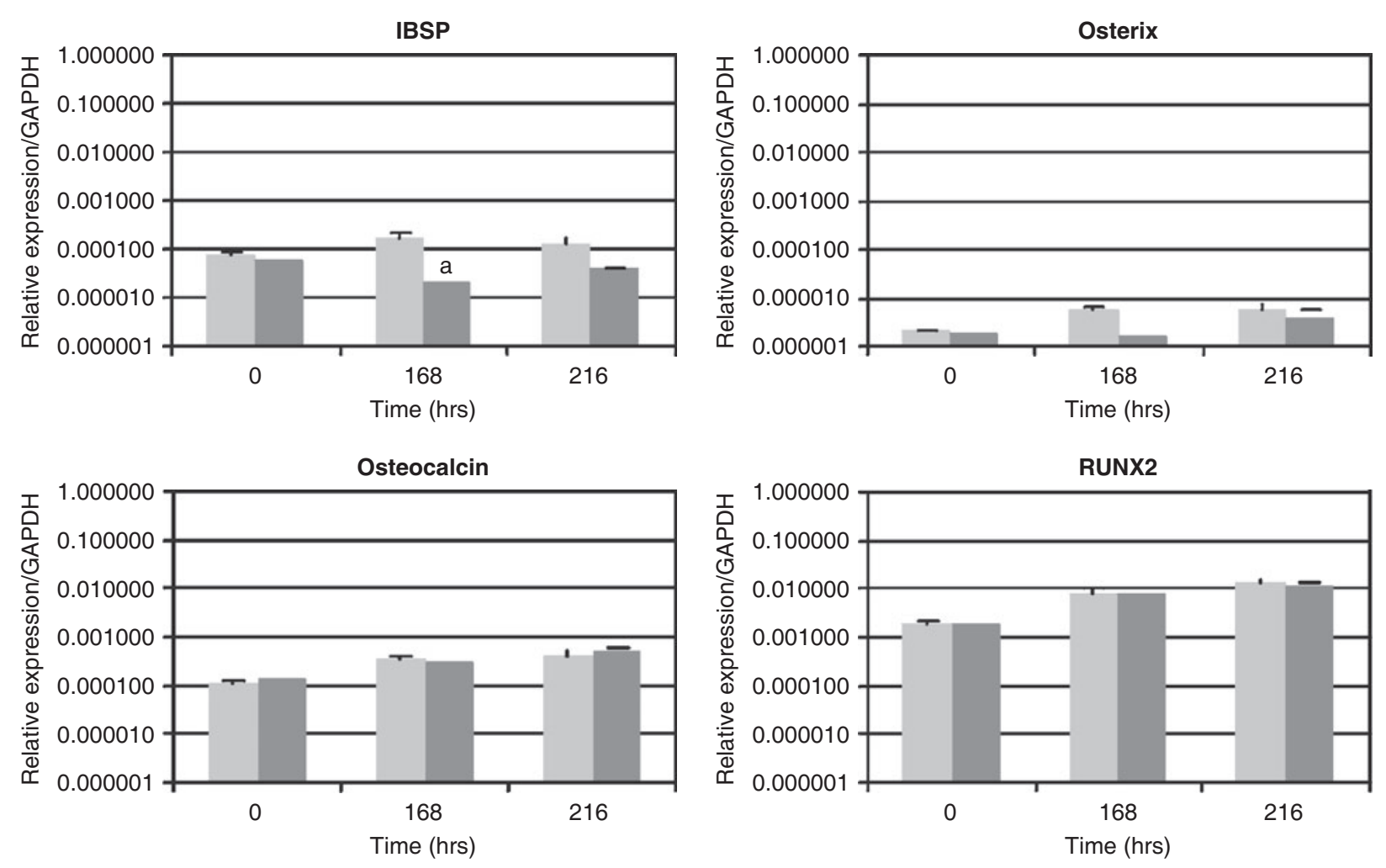

FIG. 3. Expression of osteoblastic markers during osteogenic differentiation of hsa-miR-135b-overexpressing unrestricted somatic stem cells (USSCs). A real-time RT-PCR analysis of RUNX2, bone sialoprotein (IBSP), Osteocalcin, and Osterix expression relative to GAPDH was performed at indicated time points. Light gray columns indicate empty vector-transduced USSCs (control) and dark gray columns hsa-miR-135b-overexpressing USSCs. Data represent mean of 2 amplifications per each condition, with standard error shown. Statistically significant data, relative to $t=0$ shown with $P$ value $\leq 0.05$ (marked as "a").

target genes such as CADM3 and GPR12 encode, respectively, for the cell adhesion molecule and G-protein-coupled highaffinity receptor for sphingosylphosphorylcholine (SPC). SPC has been reported to induce proliferation of human adipose tissue-derived MSCs [37]. Therefore, one could speculate that the down-regulation of GPR12 in osteogenesis of hsa-miR135b-overexpressing USSCs may be associated with declined proliferation stimulatory activity of SPC in the osteogenic differentiation of USSCs.

The most interesting experimental target gene appeared to be COL15A1. COL15A1 was recently described as a new and the most up-regulated gene in isolated human osteoblasts and osteoblasts differentiated from human bone marrow-derived mesenchymal stem cells (MSCs) [38]. Importantly, this osteoblast-secreted collagen was found not only to be localized in the new bone tissue forming area but was also downregulated by free ionized extracellular calcium. Taking into account that at the mineralization stage a decrease in free ionized extracellular calcium occurs [39], this might indicate an involvement of COL15A1 as an osteoblast-secreted and calcium-regulated protein in the mineralization process of USSCs as previously suggested for human bone marrowderived MSCs [38].

\section{Acknowledgments}

We would like to thank Ester Piek for critical reading of the manuscript and initial support with differentiation protocols and experiments. And we thank Karen Mattheisen for the native English speaker correction of the manuscript. This study was supported by DPTE grant NGC6719.

\section{Author Disclosure Statement}

All authors have no conflicts of interest.

\section{References}

1. Lee Y, K Jeon, JT Lee, S Kim and VN Kim. (2002). MicroRNA maturation: stepwise processing and subcellular localization. EMBO J 21:4663-4670.

2. Kim VN. (2005a). MicroRNA biogenesis: coordinated cropping and dicing. Nat Rev Mol Cell Biol 6:376-385.

3. Kim VN. (2005b). Small RNAs: classification, biogenesis, and function. Mol Cells 19:1-15.

4. Song L and RS Tuan. (2006). MicroRNAs and cell differentiation in mammalian development. Birth Defects Res C Embryo Today 78:140-149.

5. Jovanovic M and MO Hengartner. (2006). miRNAs and apoptosis: RNAs to die for. Oncogene 25:6176-6187.

6. Zhang B, Q Wang and X Pan. (2007). MicroRNAs and their regulatory roles in animals and plants. J Cell Physiol 210:279-289.

7. Yekta S, IH Shih and DP Bartel. (2004). MicroRNA-directed cleavage of HOXB8 mRNA. Science 304:594-596.

8. Yoon S and G De Micheli. (2006). Computational identification of microRNAs and their targets. Birth Defects Res C Embryo Today 78:118-128.

9. Griffiths-Jones S, RJ Grocock, S van Dongen, A Bateman and AJ Enright. (2006). miRBase: microRNA sequences, targets and gene nomenclature. Nucleic Acids Res 34(Database issue):D140-D144. 
10. Krek A, D Grün, MN Poy, R Wolf, L Rosenberg, EJ Epstein, $P$ MacMenamin, I da Piedade, KC Gunsalus, M Stoffel and N Rajewsky. (2005). Combinatorial microRNA target predictions. Nat Genet 37:495-500.

11. Kiriakidou M, PT Nelson, A Kouranov, P Fitziev, C Bouyioukos, $\mathrm{Z}$ Mourelatos and A Hatzigeorgiou. (2004). A combined computational-experimental approach predicts human microRNA targets. Genes Dev 18:1165-1178.

12. Lewis BP, CB Burge and DP Bartel. (2005). Conserved seed pairing, often flanked by adenosines, indicates that thousands of human genes are microRNA targets. Cell 120:15-20.

13. Kögler G, SSensken, JA Airey, T Trapp, M Müschen, N Feldhahn, S Liedtke, RV Sorg, J Fischer, C Rosenbaum, S Greschat, A Knipper, J Bender, O Degistirici, J Gao, AI Caplan, EJ Colletti, G AlmeidaPorada, HW Müller, E Zanjani and P Wernet. (2004). A new human somatic stem cell from placental cord blood with intrinsic pluripotent differentiation potential. J Exp Med 200:123-135.

14. Sensken S, S Waclawczyk, AS Knaupp, T Trapp, J Enczmann, P Wernet and G Kogler. (2007). In vitro differentiation of human cord blood-derived unrestricted somatic stem cells towards an endodermal pathway. Cytotherapy 9:362-378.

15. Greschat S, J Schira, P Küry, C Rosenbaum, MA de Souza Silva, G Kögler, P Wernet and HW Müller. (2008). Unrestricted somatic stem cells from human umbilical cord blood can be differentiated into neurons with a dopaminergic phenotype. Stem Cells Dev 17:221-232.

16. Rodan GA. (1992). Introduction to bone biology. Bone 13(Suppl 1):S3-S6.

17. Olsen BR, AM Reginato and W Wang. (2000). Bone development. Annu Rev Cell Dev Biol 16:191-220.

18. Gregory CA, WG Gunn, A Peister and DJ Prockop. (2004). An Alizarin red-based assay of mineralization by adherent cells in culture: comparison with cetylpyridinium chloride extraction. Anal Biochem 329:77-84.

19. Voorhoeve PM, C le Sage, M Schrier, AJ Gillis, H Stoop, R Nagel, YP Liu, J van Duijse, J Drost, A Griekspoor, E Zlotorynski, N Yabuta, G De Vita, H Nojima, LH Looijenga and R Agami. (2006). A genetic screen implicates miRNA-372 and miRNA-373 as oncogenes in testicular germ cell tumors. Cell 124:1169-1181.

20. Chen C, DA Ridzon, AJ Broomer, Z Zhou, DH Lee, JT Nguyen, M Barbisin, NL Xu, VR Mahuvakar, MR Andersen, KQ Lao, KJ Livak and KJ Guegler. (2005). Real-time quantification of microRNAs by stem-loop RT-PCR. Nucleic Acids Res 33:e179.

21. Huang W, S Yang, J Shao and YP Li. (2007). Signaling and transcriptional regulation in osteoblast commitment and differentiation. Front Biosci 12:3068-3092.

22. Li Z, MQ Hassan, $S$ Volinia, AJ van Wijnen, JL Stein, CM Croce, JB Lian and GS Stein. (2008). A microRNA signature for a BMP2induced osteoblast lineage commitment program. Proc Natl Acad Sci USA 105:13906-13911.

23. Landgraf $\mathrm{P}, \mathrm{M}$ Rusu, R Sheridan, A Sewer, N Iovino, A Aravin, $\mathrm{S}$ Pfeffer, A Rice, AO Kamphorst, M Landthaler, C Lin, ND Socci, L Hermida, V Fulci, S Chiaretti, R Foà, J Schliwka, U Fuchs, A Novosel, RU Müller, B Schermer, U Bissels, J Inman, Q Phan, M Chien, DB Weir, R Choksi, G De Vita, D Frezzetti, HI Trompeter, V Hornung, G Teng, G Hartmann, M Palkovits, R Di Lauro, P Wernet, G Macino, CE Rogler, JW Nagle, J Ju, FN Papavasiliou, T Benzing, P Lichter, W Tam, MJ Brownstein, A Bosio, A Borkhardt, JJ Russo, C Sander, M Zavolan and T Tuschl. (2007). A mammalian microRNA expression atlas based on small RNA library sequencing. Cell 129:1401-1414.

24. Oskowitz AZ, J Lu, P Penfornis, J Ylostalo, J McBride, EK Flemington, DJ Prockop and R Pochampally. (2008). Human multipotent stromal cells from bone marrow and microRNA: regulation of differentiation and leukemia inhibitory factor expression. Proc Natl Acad Sci USA 105:18372-18377.

25. Whyte MP. (1994). Hypophosphatasia and the role of alkaline phosphatase in skeletal mineralization. Endocr Rev 15:439-461.

26. Anderson HC, HH Hsu, DC Morris, KN Fedde and MP Whyte. (1997). Matrix vesicles in osteomalacic hypophosphatasia bone contain apatite-like mineral crystals. Am J Pathol 151:1555-1561.

27. Narisawa S, N Fröhlander and JL Millán. (1997). Inactivation of two mouse alkaline phosphatase genes and establishment of a model of infantile hypophosphatasia. Dev Dyn 208:432-446.

28. Wennberg C, L Hessle, P Lundberg, S Mauro, S Narisawa, UH Lerner and JL Millán. (2000). Functional characterization of osteoblasts and osteoclasts from alkaline phosphatase knockout mice. J Bone Miner Res 15:1879-1888.

29. Anderson HC, JB Sipe, L Hessle, R Dhanyamraju, E Atti, NP Camacho, JL Millán and R Dhamyamraju. (2004). Impaired calcification around matrix vesicles of growth plate and bone in alkaline phosphatase-deficient mice. Am J Pathol 164:841-847.

30. Orimo $\mathrm{H}$ and T Shimada. (2008). The role of tissue-nonspecific alkaline phosphatase in the phosphate-induced activation of alkaline phosphatase and mineralization in SaOS-2 human osteoblast-like cells. Mol Cell Biochem 315:51-60.

31. Waymire KG, JD Mahuren, JM Jaje, TR Guilarte, SP Coburn and GR MacGregor. (1995). Mice lacking tissue non-specific alkaline phosphatase die from seizures due to defective metabolism of vitamin B-6. Nat Genet 11:45-51.

32. Malaval L, NM Wade-Guéye, M Boudiffa, J Fei, R Zirngibl, F Chen, N Laroche, JP Roux, B Burt-Pichat, F Duboeuf, G Boivin, P Jurdic, MH Lafage-Proust, J Amédée, L Vico, J Rossant and JE Aubin. (2008). Bone sialoprotein plays a functional role in bone formation and osteoclastogenesis. J Exp Med 205:1145-1153.

33. Ogata Y. (2008). Bone sialoprotein and its transcriptional regulatory mechanism. J Periodont Res 43:127-135.

34. Marson A, SS Levine, MF Cole, GM Frampton, T Brambrink, S Johnstone, MG Guenther, WK Johnston, M Wernig, J Newman, JM Calabrese, LM Dennis, TL Volkert, S Gupta, J Love, N Hannett, PA Sharp, DP Bartel, R Jaenisch and RA Young. (2008). Connecting microRNA genes to the core transcriptional regulatory circuitry of embryonic stem cells. Cell 134:521-533.

35. Bartel DP and CZ Chen. (2004). Micromanagers of gene expression: the potentially widespread influence of metazoan microRNAs. Nat Rev Genet 5:396-400.

36. Krützfeldt J, N Rajewsky, R Braich, KG Rajeev, T Tuschl, M Manoharan and M Stoffel. (2005). Silencing of microRNAs in vivo with "antagomirs." Nature 438:685-689.

37. Jeon ES, HY Song, MR Kim, HJ Moon, YC Bae, JS Jung and JH Kim. (2006). Sphingosylphosphorylcholine induces proliferation of human adipose tissue-derived mesenchymal stem cells via activation of JNK. J Lipid Res 47:653-664.

38. Lisignoli G, K Codeluppi, K Todoerti, C Manferdini, A Piacentini, N Zini, F Grassi, L Cattini, R Piva, V Rizzoli, A Facchini, N Giuliani and A Neri. (2009). Gene array profile identifies collagen type XV as a novel human osteoblast-secreted matrix protein. J Cell Physiol 220:401-409.

39. Dvorak MM, A Siddiqua, DT Ward, DH Carter, SL Dallas, EF Nemeth and D Riccardi. (2004). Physiological changes in extracellular calcium concentration directly control osteoblast function in the absence of calciotropic hormones. Proc Natl Acad Sci USA 101:5140-5145.

$$
\begin{array}{r}
\text { Address correspondence to: } \\
\text { Dr. Joop H. Jansen } \\
\text { Central Hematology Laboratory } \\
\text { Molecular Hemato-Oncology Unit } \\
\text { Radboud University Nijmegen Medical Centre } \\
\text { Nijmegen Centre for Molecular Life Sciences } \\
\text { P.O. Box } 9101 \\
\text { Nijmegen } 6500 \mathrm{HB} \\
\text { The Netherlands } \\
\text { E-mail: j.jansen@chl.umcn.nl }
\end{array}
$$

Received for publication April 1, 2009

Accepted after revision October 1, 2009

Prepublished on Liebert Instant Online October 1, 2009 
